\title{
MOTIVIC COHOMOLOGY OVER DEDEKIND RINGS
}

\author{
THOMAS GEISSER*
}

\begin{abstract}
We study properties of Bloch's higher Chow groups on smooth varieties over Dedekind rings. We prove a conditional Gersten resolution, which implies that $\mathcal{H}^{i}(\mathbb{Z}(n))=0$ for $i>n$ and that there is a Gersten resolution for $\mathcal{H}^{i}\left(\mathbb{Z} / p^{r}(n)\right)$, if the residue characteristic is $p$. We also show that the Bloch-Kato conjecture implies the Beilinson-Lichtenbaum conjecture, an identification $\mathbb{Z} / m(n)_{\text {ét }} \cong \mu_{m}^{\otimes n}$, for $m$ invertible on the scheme, and a Gersten resolution with (arbitrary) finite coefficients. Over a discrete valuation ring of mixed characteristic $(0, p)$, we construct a map from motivic cohomology to syntomic cohomology, which is a quasi-isomorphism provided the Bloch-Kato conjecture holds.
\end{abstract}

\section{INTRODUCTION}

Let $X$ be an (essentially) smooth variety over a base $B$, and define motivic cohomology $H^{*}(X, \mathbb{Z}(n))$ and étale motivic cohomology $H_{\text {êt }}^{*}(X, \mathbb{Z}(n))$ as the hypercohomology of Bloch's cycle complex $\mathbb{Z}(n)$ for the Zariski and étale topology, respectively. If $B$ is the spectrum of a field, then these groups agree with the cohomology of Bloch's cycle complex on the one hand, and with Voevodsky's motivic cohomology groups on the other hand. In case $B$ is a discrete valuation ring, Levine $[16,15]$ has established several properties, most notably the localization property, which assures that cohomology and hypercohomology of $\mathbb{Z}(n)$ agree. The purpose of this article is to establish further sheaf theoretic properties of $\mathbb{Z}(n)$ if the base is a Dedekind ring. Fix an essentially smooth scheme $X$ over the spectrum of a Dedekind ring $B$. We recall basic properties and the results of Levine, and show the following conditional Gersten resolution:

Theorem 1.1. Assume that for any discrete valuation ring $R$, essentially of finite type over $B$, with quotient field $K$, the map $H^{s}(R, \mathbb{Z}(n)) \rightarrow H^{s}(K, \mathbb{Z}(n))$ is injective. Then there is an exact sequence of Zariski sheaves on $X$,

$$
\begin{aligned}
0 \rightarrow \mathcal{H}^{s}\left(\mathbb{Z}(n)_{\mathrm{Zar}}\right) \rightarrow \bigoplus_{x \in X^{(0)}}\left(i_{x}\right)_{*} H^{s} & (k(x), \mathbb{Z}(n)) \\
& \rightarrow \bigoplus_{x \in X^{(1)}}\left(i_{x}\right)_{*} H^{s-1}(k(x), \mathbb{Z}(n-1)) \rightarrow \cdots .
\end{aligned}
$$

The analogous statement holds with arbitrary coefficients. The proof is based on the modification of Quillen's original argument [21] by Gillet-Levine [9]. As corollaries, we obtain that the complex $\mathbb{Z}(n)$ is acyclic in degrees above $n$, and a Gersten resolution for $\mathcal{H}^{s}\left(\mathbb{Z} / p^{r}(n)\right)$ if $B$ has residue characteristic $p$.

\footnotetext{
* Supported in part by JSPS, NSF, and the Alfred P. Sloan Foundation.
} 
For étale motivic cohomology, there is no localization long exact sequence. Instead one expects for $i: Y \rightarrow X$ the inclusion of one of the closed fibers a quasiisomorphism

$$
\mathbb{Z}(n-1)_{\text {ét }}[-2] \stackrel{\sim}{\longrightarrow} \tau_{\leq n+1} R i^{!} \mathbb{Z}(n)_{\text {ét }} .
$$

The Bloch-Kato conjecture [3] states that for any field $F$, and $m$ relatively prime to the characteristic of $F$, the symbol map from Milnor $K$-theory to Galois cohomology,

$$
K_{n}^{M}(F) / m \rightarrow H_{\text {ét }}^{n}\left(F, \mu_{m}^{\otimes n}\right)
$$

is surjective. The conjecture is known for $m$ a power of 2 by Voevodsky [27].

Theorem 1.2. The Bloch-Kato conjecture implies the following:

(1) (Purity) The quasi-isomorphism (1) holds.

(2) (Beilinson-Lichtenbaum) There is a quasi-isomorphism

$$
\mathbb{Z}(n)_{\mathrm{Zar}} \stackrel{\sim}{\longrightarrow} \tau_{\leq n+1} R \epsilon_{*} \mathbb{Z}(n)_{\text {ét }}
$$

(3) (Rigidity) For an essentially smooth Henselian local ring $R$ over $B$ with residue field $k$ and $\frac{1}{m} \in k$,

$$
H^{i}(R, \mathbb{Z} / m(n)) \cong H^{i}(k, \mathbb{Z} / m(n)) .
$$

(4) (Étale sheaf) If $\frac{1}{m} \in \mathcal{O}^{\times}(B)$, then there is a quasi-isomorphism of étale sheaves

$$
\mathbb{Z} / m(n)_{\text {ét }} \cong \mu_{m}^{\otimes n} .
$$

(5) (Gersten resolution) For any $m$, there is an exact sequence:

$$
\begin{aligned}
0 \rightarrow \mathcal{H}^{s}\left(\mathbb{Z} / m(n)_{\mathrm{Zar}}\right) & \rightarrow \bigoplus_{x \in X^{(0)}}\left(i_{x}\right)_{*} H^{s}(k(x), \mathbb{Z} / m(n)) \\
& \rightarrow \bigoplus_{x \in X^{(1)}}\left(i_{x}\right)_{*} H^{s-1}(k(x), \mathbb{Z} / m(n-1)) \longrightarrow \cdots
\end{aligned}
$$

Note that the combination of (2), (4) and (5) gives a Gersten resolution for the sheaf $R^{s} \epsilon_{*} \mu_{m}^{\otimes n}$ for $s \leq n, m$ invertible on $B$ and $\epsilon: X_{\text {ét }} \rightarrow X_{\text {Zar }}$ the change of site map.

If $X$ is a smooth scheme over a discrete valuation ring of characteristic $(0, p)$, then (1) implies the existence of a distinguished triangle

$$
i^{*} \mathbb{Z} / p^{r}(n)_{\text {ét }} \rightarrow \tau_{\leq n} i^{*} R j_{*} \mu_{p^{r}}^{\otimes n} \rightarrow \nu_{r}^{n-1}[-n] .
$$

By results of Kato and Kurihara [13], this property is shared by syntomic cohomology $S_{r}(n)$ of Fontaine-Messing [6], whenever the latter is defined. In general, we define $S_{r}(n)$ as the cone of a map $\kappa: \tau_{\leq n} i^{*} R j_{*} \mu_{p^{r}}^{\otimes n} \rightarrow \nu_{r}^{n-1}[-n]$ defined by Kato. The following theorem shows that motivic cohomology can be thought of as a generalization of syntomic cohomology, see Milne [18, remark 2.7] and Schneider $[23]$.

Theorem 1.3. There is a unique map $i^{*} \mathbb{Z} / p^{r}(n)$ ét $\rightarrow S_{r}(n)$ in the derived category of étale sheaves on $Y$ which is compatible with the maps of both complexes to $\tau_{\leq n} i^{*} R j_{*} \mu_{p^{r}}^{\otimes n}$. The map is a quasi-isomorphism provided that the Bloch-Kato conjecture with mod p-coefficients holds. 
This can be used to better understand the relationship between various proofs of the crystalline conjecture, and to construct cycle maps to syntomic cohomology.

Notation: Unless otherwise stated, $B$ is the spectrum of a Dedekind ring $D$, and $X$ an essentially smooth scheme over $B$. For a point $b \in B$ we denote by $i_{b}: X_{b} \rightarrow X$ the closed embedding of the fiber of $X$ over $b$, and by $\tilde{X}_{b}$ the fiber over the localization of $B$ at $b$. We let $X_{\text {ét }}, X_{\mathrm{Nis}}$ and $X_{\mathrm{Zar}}$ be the category of étale schemes over $X$ equipped with the étale, Nisnevich and Zariski topology. These sites are noetherian, in particular cohomology commutes with filtered colimits. In case the site has infinite cohomological dimension (which may happen for the étale topology), we calculate derived functors of unbounded complexes using the method of Spaltenstein [24]. The maps $X_{\text {ét }} \stackrel{\alpha}{\longrightarrow} X_{\text {Nis }} \stackrel{\beta}{\longrightarrow} X_{\text {Zar }}$ and $\epsilon=\beta \circ \alpha$ are the canonical maps of sites. Clearly, $\epsilon^{*}, \alpha^{*}, \beta^{*}$ are exact and preserve colimits, whereas $\epsilon_{*}, \alpha_{*}, \beta_{*}$ are left exact, preserve injectives and limits.

Acknowledgements: This paper relies heavily on the work of M. Levine and discussions with him. Discussions with W. Niziol and K. Sato have been helpful. Finally, I would like to thank T. Saito and the Tokyo University Department of Mathematics for their hospitality.

\section{Some homological ALGEBRA}

Let $i: Y \rightarrow X$ be a closed subscheme with open complement $j: U \rightarrow X$. There are mutually adjoint functors $i^{*} \vdash i_{*} \vdash i^{!}$and $j_{!} \vdash j^{*} \vdash j_{*}$. In particular, $i^{!}, j_{*}$ are left exact, $i_{*}, j^{*}$ are exact, and in our situation $i^{*}$ and $j$ ! are exact. Consequently, $i_{*}, i^{!}, j^{*}, j_{*}$ preserve limits and injectives, whereas $i^{*}, i_{*}, j_{!}, j^{*}$ preserve colimits.

Lemma 2.1. Let $X$ be essentially of finite type over $B, j: X_{K} \rightarrow X$ be the embedding of the generic fiber. Let $C^{\cdot}$ be a complex of sheaves on $X$. Then there is a distinguished triangle

$$
\cdots \rightarrow \bigoplus_{b \in B^{(0)}} i_{b *} R i_{b}^{\vdots} C^{\cdot} \rightarrow C^{\cdot} \rightarrow R j_{*} j^{*} C^{\cdot} \rightarrow \cdots
$$

In particular, there is a long exact sequence

$$
\cdots \rightarrow \bigoplus_{b \in B^{(0)}} H_{X_{b}}^{i}\left(X, C^{\cdot}\right) \rightarrow H^{i}\left(X, C^{\cdot}\right) \rightarrow H^{i}\left(X_{K}, C^{\cdot}\right) \rightarrow \cdots
$$

Proof. It suffices to check this at stalks, in particular it suffices to check that the triangle is exact after applying $j^{*}$ and $i_{b}^{*}$ for any closed point $b \in B$. Now $j^{*}$ and $i_{b}^{*}$ commute with colimits, and $j^{*} i_{b *}=i_{c}^{*} i_{b *}=0$ for $c \neq b$. Hence applying $j^{*}$ to the triangle gives the isomorphism $j^{*} C^{\cdot} \stackrel{\sim}{\longrightarrow} j^{*} R j_{*} j^{*} C$, and applying $i_{c}^{*}$ gives the decomposition triangle

$$
\cdots \rightarrow R i_{c}^{!} C^{\cdot} \rightarrow i_{c}^{*} C^{\cdot} \rightarrow i_{c}^{*} R j_{*} j^{*} C^{\cdot} \rightarrow \cdots .
$$

The exact sequence of cohomology groups follows because on a noetherian site cohomology commutes with colimits of sheaves. 
Proposition 2.2. a) The following maps are isomorphisms of derived functors

$$
\begin{array}{rlrl}
\epsilon^{*} j^{*} & \stackrel{\sim}{\longrightarrow} j^{*} \epsilon^{*} & R \epsilon_{*} R j_{*} & \stackrel{\sim}{\longrightarrow} R j_{*} R \epsilon_{*} \\
\epsilon^{*} i^{*} & \stackrel{\sim}{\sim} i^{*} \epsilon^{*} & R \epsilon_{*} i_{*} & \stackrel{\sim}{\longrightarrow} i_{*} R \epsilon_{*} \\
\epsilon^{*} i_{*} & \stackrel{\sim}{\longrightarrow} i_{*} \epsilon^{*} & R \epsilon_{*} R i^{!} & \stackrel{\sim}{\longrightarrow} R i ! R \epsilon_{*} \\
\epsilon^{*} j ! & \stackrel{\sim}{\longrightarrow} j ! \epsilon^{*} & R \epsilon_{*} j^{*} & \stackrel{\sim}{\longrightarrow} j^{*} R \epsilon_{*}
\end{array}
$$

The analogous statements for $\alpha$ and $\beta$ hold.

b) If the étale cohomological dimension of each residue field of $Y$ is finite, then

$$
i^{*} R \alpha_{*} \stackrel{\sim}{\longrightarrow} R \alpha_{*} i^{*} .
$$

Proof. a) The maps on the left arise by the universal property of sheafification. The statements on the right are adjoint to the statements on the left, and it suffices to verify one of each pair. Since all functors involved in the right hand side preserve injectives, it suffices to verify the isomorphisms of functors before deriving. The statements $\epsilon^{*} j^{*} \cong j^{*} \epsilon^{*}$ and $\epsilon_{*} i_{*} \cong i_{*} \epsilon_{*}$ are obvious. The other two statements follow because

$$
\left(\epsilon^{*} i_{*} \mathcal{F}\right)_{\bar{x}} \cong\left(i_{*} \epsilon^{*} \mathcal{F}\right)_{\bar{x}} \cong \begin{cases}\mathcal{F}_{\bar{x}} & x \in Y \\ 0 & x \notin Y,\end{cases}
$$

and similarly

$$
\left(\epsilon^{*} j ! \mathcal{F}\right)_{\bar{x}} \cong\left(j ! \epsilon^{*} \mathcal{F}\right)_{\bar{x}} \cong \begin{cases}\mathcal{F}_{\bar{x}} & x \in U \\ 0 & x \notin U\end{cases}
$$

b) Let $(R, \mathfrak{m})$ be a Henselian local ring and $I \subseteq \mathfrak{m}$ an ideal of $R$. Since the closed embedding $\operatorname{Spec} R / I \stackrel{i}{\rightarrow} \operatorname{Spec} R$ induces an equivalence of categories $(\operatorname{Spec} R)_{\text {ét }} \stackrel{i^{*}}{\longrightarrow}$ $(\operatorname{Spec} R / I)_{\text {ét }}$, the restriction map $H_{\text {êt }}^{s}(R, \mathcal{F}) \stackrel{i^{*}}{\longrightarrow} H_{\text {ét }}^{s}\left(R / I, i^{*} \mathcal{F}\right)$ is an isomorphism for any sheaf $\mathcal{F}$. By the usual spectral sequence argument, the same holds for complexes of sheaves if the étale cohomological dimension of $\operatorname{Spec} R / \mathfrak{m}$ is finite.

Now let $y \in Y \subseteq X$ and $\mathcal{F}$ be a complex of étale sheaves on $X, R=\mathcal{O}_{X, y}^{h}$ be the Henselian local ring of $y$ in $X$ and $R / I=\mathcal{O}_{Y, y}^{h}$ be the Henselian local ring of $y$ in $Y$. We get

$$
\begin{aligned}
& \left(i^{*} R^{s} \alpha_{*} \mathcal{F}^{\cdot}\right)_{y}=H_{\mathrm{Nis}}^{s}\left(R / I, i^{*} R \alpha_{*} \mathcal{F}^{*}\right) \cong H_{\mathrm{Nis}}^{s}\left(R, R \alpha_{*} \mathcal{F}^{*}\right) \\
& \quad \cong H_{\mathrm{et}}^{s}\left(R, \mathcal{F}^{*}\right) \cong H_{\mathrm{et}}^{s}\left(R / I, i^{*} \mathcal{F}^{*}\right) \cong H_{\mathrm{Nis}}^{s}\left(R / I, R \alpha_{*} i^{*} \mathcal{F}^{*}\right)=\left(R^{s} \alpha_{*} i^{*} \mathcal{F}^{*}\right)_{y}
\end{aligned}
$$

Remark. The other combinations of the functors do not commute in general. For example, if $R$ is a local ring with closed subscheme $i: \operatorname{Spec} R / I \rightarrow R$, and if $R_{I}^{h}$ is the Henselization of $R$ along $I$, then

$$
\begin{aligned}
& H_{\mathrm{Zar}}^{s}\left(R / I, i^{*} R \epsilon_{*} \mathcal{F}\right) \cong H_{\mathrm{Zar}}^{s}\left(R, R \epsilon_{*} \mathcal{F}\right) \cong H_{\mathrm{et}}^{s}(R, \mathcal{F}) \\
& H_{\mathrm{Zar}}^{s}\left(R / I, R \epsilon_{*} i^{*} \mathcal{F}\right) \cong H_{\mathrm{et}}^{s}\left(R / I, i^{*} \mathcal{F}\right)=H_{\mathrm{et}}^{s}\left(R_{I}^{h}, \mathcal{F}\right)
\end{aligned}
$$

The failure of the adjoint maps $\epsilon^{*} R j_{*} \rightarrow R j_{*} \epsilon^{*}$ and $\epsilon^{*} R i^{!} \rightarrow R i^{!} \epsilon^{*}$ to be quasiisomorphisms are closely related to the Beilinson-Lichtenbaum conjecture. 


\section{Motivic Cohomology}

We define motivic cohomology as the the hypercohomology of Bloch's cycle complex: Let $z^{n}(X, i)$ be the free abelian group on closed integral subschemes of codimension $n$ on $X \times \Delta^{i}$ which intersect all faces properly. Intersecting with faces defines a structure of a simplicial abelian group, and hence gives a complex $z^{n}(X, *)$. The complex $z^{n}(X, *)$ is easily seen to be covariant for proper morphisms (with the appropriate shift in weight and degree) and contravariant for flat morphisms.

Lemma 3.1. Let $X$ be quasi-compact. The presheaves $z^{n}(-, i)$ are sheaves on the site $(f t / X)_{f l}$, the category of schemes of finite type over $X$, with flat morphisms of finite type as coverings.

Proof. Every scheme of finite type over a quasi-compact scheme is again quasicompact. Since flat maps of finite type are open, the site $(f t / X)_{f l}$ is noetherian. To check the sheaf property, we can restrict ourselves to coverings of $X$ which consist of a faithfully flat morphism $Y \rightarrow X$ by [19, prop. 3.5], and we have to show that the following sequence is exact:

$$
0 \rightarrow z^{n}(X, i) \rightarrow z^{n}(Y, i) \stackrel{p_{1}^{*}-p_{2}^{*}}{\longrightarrow} z^{n}\left(Y \times_{X} Y, i\right) .
$$

Exactness on the left is clear, and exactness in the middle is the flat descent theorem, [19, I 2.23].

The complex of sheaves $\mathbb{Z}(n)_{t}$ on the site $X_{t}$, where $t \in\{$ Zar, Nis, et $\}$, is defined as the cohomological complex with $z^{n}(-, 2 n-i)$ in degree $i$. For an abelian group $A$ we define $A(n)$ to be $\mathbb{Z}(n) \otimes A$. Since $\mathbb{Z}(n)$ is a complex of sheaves for the flat topology,

$$
\epsilon^{*} \mathbb{Z}(n)_{\text {Zar }}=\mathbb{Z}(n)_{\text {ét }}, \quad \epsilon_{*} \mathbb{Z}(n)_{\text {ét }}=\mathbb{Z}(n)_{\text {Zar }},
$$

and similarly for $\alpha^{*} \vdash \alpha_{*}$ and $\beta^{*} \vdash \beta_{*}$. Consequently,

$$
\epsilon^{*} i^{!} \mathbb{Z}(n)_{\mathrm{Zar}} \cong i^{!} \epsilon^{*} \mathbb{Z}(n)_{\mathrm{Zar}} \quad \epsilon^{*} j_{*} \mathbb{Z}(n)_{\mathrm{Zar}} \cong j_{*} \epsilon^{*} \mathbb{Z}(n)_{\mathrm{Zar}} .
$$

We define $H_{t}^{i}(X, \mathbb{Z}(n))$ as the hypercohomology of $\mathbb{Z}(n)_{t}$ and often omit $t$ if $t=$ Zar. A flat map $f: X \rightarrow Y$ induces via the pull back of cycles a map of sheaves $f^{*} \mathbb{Z}(n)^{Y} \rightarrow \mathbb{Z}(n)^{X}$. Together with the adjoint map we get $\mathbb{Z}(n)^{Y} \rightarrow$ $R f_{*} f^{*} \mathbb{Z}(n)^{Y} \rightarrow R f_{*} \mathbb{Z}(n)^{X}$, and hence

$$
H_{t}^{i}(Y, \mathbb{Z}(n)) \rightarrow H_{t}^{i}\left(Y, R f_{*} \mathbb{Z}(n)\right) \stackrel{\cong}{\rightrightarrows} H_{t}^{i}(X, \mathbb{Z}(n)) .
$$

Theorem 3.2. (Levine) Let $X$ be essentially of finite type over a discrete valuation ring. If $i: Z \rightarrow X$ a closed subscheme of codimension $c$ with open complement $j: U \rightarrow X$, then the exact sequence

$$
0 \rightarrow z^{q-c}(Z, *) \stackrel{i_{*}}{\longrightarrow} z^{q}(X, *) \stackrel{j^{*}}{\longrightarrow} z^{q}(U, *)
$$

forms a distinguished triangle in the derived category of abelian groups. In particular, motivic cohomology $H_{\mathrm{Zar}}^{s}(X, \mathbb{Z}(n))$ agrees with the cohomology of the complex of abelian groups $\Gamma(X, \mathbb{Z}(n))$.

Proof. The first statement is [16, Theorem 1.5]. For the second statement, we employ Thomason's criterion [26, Prop. 2.3]. Let $\mathbb{Z}(n) \stackrel{\eta}{\rightarrow} I^{*}$ be an injective resolution (for the Zariski topology). Then $\eta$ is an isomorphism on stalks. On the other hand, localization implies that the cohomology of $\mathbb{Z}(n)$ satisfies the Mayer-Vietoris property, whereas hypercohomology, i.e. the cohomology of $I^{*}$, automatically has this 
property. Hence $\eta$ induces a quasi-isomorphism on global sections.

Corollary 3.3. Let $X \stackrel{p}{\rightarrow} B$ be essentially of finite type. If $i: Z \rightarrow X$ is a closed subscheme of pure codimension $c$ with open complement $j: U \rightarrow X$, then the exact sequence

$$
0 \rightarrow i_{*} \mathbb{Z}(n-c)^{Z}[-2 c] \rightarrow \mathbb{Z}(n)^{X} \rightarrow j_{*} \mathbb{Z}(n)^{U}
$$

forms a distinguished triangle in the derived category of sheaves on $X$. In particular, there is an isomorphism of hypercohomology groups

$$
H_{\mathrm{Zar}}^{s}\left(B, p_{*} \mathbb{Z}(n)\right) \cong H_{\mathrm{Zar}}^{s}(X, \mathbb{Z}(n))=H_{\mathrm{Zar}}^{s}\left(B, R p_{*} \mathbb{Z}(n)\right) .
$$

Proof. We verify the exactness stalkwise. Since the cycle complex commutes with direct limits of rings, it suffices to verify the statement for the local ring at a point $x \in X$. If $x$ is in the generic fiber, then $\mathcal{O}_{X, x}$ is essentially of finite type over a field, the situation which was considered by Bloch [1]. If $x$ is in a closed fiber, then $\mathcal{O}_{X, x}$ satisfies the hypothesis of the theorem.

To see that the adjoint map $p_{*} \mathbb{Z}(n) \rightarrow R p_{*} \mathbb{Z}(n)$ is a quasi-isomorphism, consider the stalk at $b \in B$. Then by the theorem,

$$
\mathcal{H}^{s}\left(p_{*} \mathbb{Z}(n)\right)_{b} \cong H^{s}\left(\Gamma\left(\tilde{X}_{b}, \mathbb{Z}(n)\right)\right) \cong H_{\text {Zar }}^{s}\left(\tilde{X}_{b}, \mathbb{Z}(n)\right)=R^{s} p_{*} \mathbb{Z}(n)_{b} .
$$

Remark. By the same argument we get a quasi-isomorphism $(p \circ j)_{*} \mathbb{Z}(n)^{U} \cong R(p \circ$ $j)_{*} \mathbb{Z}(n)^{U}$, and after applying $p^{*}$, a quasi-isomorphism

$$
j_{*} \mathbb{Z}(n)_{\mathrm{Zar}} \stackrel{\sim}{\longrightarrow} R j_{*} \mathbb{Z}(n)_{\mathrm{Zar}} .
$$

On the other hand,

$$
\mathbb{Z}(n-c)_{\mathrm{Zar}}[-2 c] \stackrel{\sim}{\longrightarrow} i ! \mathbb{Z}(n)_{\mathrm{Zar}} \stackrel{\sim}{\longrightarrow} R i^{!} \mathbb{Z}(n)_{\mathrm{Zar}},
$$

because all complexes are quasi-isomorphic to the cone of the morphism $\mathbb{Z}(n)^{X} \rightarrow$ $j_{*} \mathbb{Z}(n)^{U}$. In particular, we can identify the canonical maps

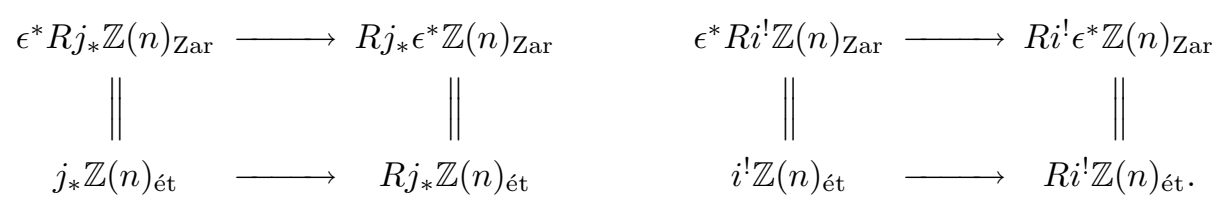

Corollary 3.4. Let $X$ be essentially of finite type over B. Then there is an exact triangle

$$
\cdots \rightarrow \bigoplus_{b \in B^{(0)}} i_{b *} \mathbb{Z}(n-1)_{\mathrm{Zar}} \rightarrow \mathbb{Z}(n)_{\mathrm{Zar}} \rightarrow R j_{*} \mathbb{Z}(n)_{\mathrm{Zar}} \rightarrow \cdots
$$

Proof. This is (5) applied to lemma 2.1.

Corollary 3.5. Let $X$ be essentially of finite type over $B$ and $\mathbb{A}_{X}^{1} \stackrel{p}{\rightarrow} X$ be the projection map. Then the pull back map

$$
H^{s}(X, \mathbb{Z}(n)) \stackrel{p^{*}}{\longrightarrow} H^{s}\left(\mathbb{A}_{X}^{1}, \mathbb{Z}(n)\right)
$$

is an isomorphism. In particular, the two maps

$$
i_{0}^{*}, i_{1}^{*}: H^{s}\left(\mathbb{A}_{X}^{1}, \mathbb{Z}(n)\right) \rightarrow H^{s}(X, \mathbb{Z}(n))
$$


induced by the zero- and one-section are defined and agree.

Proof. Because $R p_{*}$ commutes with direct sums on a noetherian site [19, III 3.6d], the localization sequence (6) reduces the statement $\mathbb{Z}(n) \cong R p_{*} \mathbb{Z}(n)$ to the field case, which was proved by Bloch [1]. Since $p^{*}$ is an isomorphism, any cycle in $z^{n}\left(\mathbb{A}_{X}^{1}, i\right)$ is equivalent to a cycle of the form $\mathbb{A}^{1} \times Z$ for $Z$ a cycle in $z^{n}(X, i)$. Now $i_{0}^{*}$ and $i_{1}^{*}$ are defined and agree for such cycles.

Since proper maps are stable under base-change, a proper map $f: X \rightarrow Y$ of relative dimension $c$ induces a map $f_{*} \mathbb{Z}(n)_{t}^{X} \rightarrow \mathbb{Z}(n-c)_{t}^{Y}[-2 c]$. Composing with the push-forward along the structure morphism $q: Y \rightarrow B$ we get a map of complexes on $B, p_{*} \mathbb{Z}(n)_{t}^{X} \rightarrow q_{*} \mathbb{Z}(n-c)_{t}^{Y}[-2 c]$, hence in view of corollary 3.3 we get covariant functoriality

$$
H^{i}(X, \mathbb{Z}(n)) \rightarrow H^{i-2 c}(Y, \mathbb{Z}(n-c)) .
$$

For the étale topology we get covariant functoriality if $f_{*} \cong R f_{*}$, for example if $f_{*}$ is exact:

$$
H_{\text {ét }}^{i}(X, \mathbb{Z}(n)) \stackrel{\sim}{\longrightarrow} H_{\text {ét }}^{i}\left(Y, R f_{*} \mathbb{Z}(n)\right) \stackrel{\sim}{\longleftarrow} H_{\text {ét }}^{i}\left(Y, f_{*} \mathbb{Z}(n)\right) \rightarrow H_{\text {ét }}^{i-2 c}(Y, \mathbb{Z}(n-c)) .
$$

However, there is no covariant functoriality for étale motivic cohomology satisfying a projection formula, as the following example shows: Let $m$ be invertible in $k$ and consider $k \stackrel{i}{\rightarrow} \mathbb{P}_{k}^{1} \stackrel{p}{\rightarrow} k$ the zero-section and projection. Since the diagonal and the class $\mathbb{P}_{k}^{1} \times 0+0 \times \mathbb{P}_{k}^{1}$ agree in $\mathrm{CH}^{1}\left(\mathbb{P}_{k}^{1} \times \mathbb{P}_{k}^{1}\right)$, the induced map on motivic cohomology of $\mathbb{P}_{k}^{1}$ agree, and by the standard calculation with idempotents this means that $i_{*} p_{*}+p^{*} i^{*}=\mathrm{id}$. Since $\mathbb{Z} / m(-1)_{\text {ét }}=0$, the first summand vanishes on the motivic cohomology of $H_{\text {ét }}^{2}\left(\mathbb{P}_{k}^{1}, \mathbb{Z} / m\right)$, and we would get $H_{\text {ét }}^{2}\left(\mathbb{P}_{k}^{1}, \mathbb{Z} / m\right)=H_{\text {ét }}^{2}(k, \mathbb{Z} / m)$, a contradiction.

Proposition 3.6. For any essentially smooth scheme $X$ over $B$, the following adjoint map is a quasi-isomorphism

$$
\mathbb{Z}(n)_{\mathrm{Zar}} \stackrel{\sim}{\longrightarrow} R \beta_{*} \mathbb{Z}(n)_{\mathrm{Nis}} .
$$

Proof. The proposition is known for a smooth scheme over a field, see [4]. We first show purity for the embedding of a closed fiber $i: X_{b} \rightarrow X$,

$$
\mathbb{Z}(n-1)_{\mathrm{Nis}}[-2] \stackrel{\sim}{\longrightarrow} R i^{!} \mathbb{Z}(n)_{\mathrm{Nis}} .
$$

Consider the embeddings $X_{b} \stackrel{\iota}{\rightarrow} \tilde{X}_{b} \stackrel{j}{\rightarrow} X$. It is easy to check at stalks that $\iota^{!} j^{*}=i^{!}$ and that $j^{*}$ maps injective sheaves to sheaves which are acyclic for $\iota^{!}$. Hence we can localize the base at $b$. Comparing $\beta^{*} i_{\text {Zar }}^{*}$ applied to (6) with $i_{\text {Nis }}^{*}$ applied to (3) via the adjoint map, we get the diagram

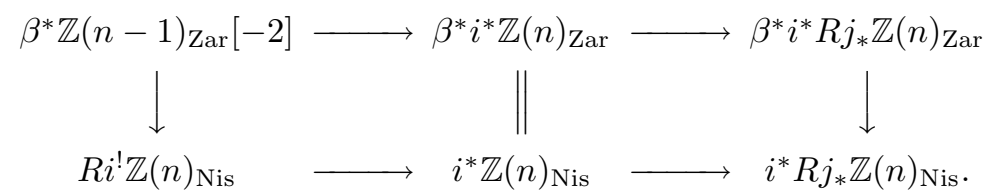

The right hand map hence the left hand map is a quasi-isomorphism because the proposition is known over a field,

$$
\beta^{*} R j_{*} \mathbb{Z}(n)_{\mathrm{Zar}} \stackrel{\sim}{\longrightarrow} \beta^{*} R j_{*} R \beta_{*} \mathbb{Z}(n)_{\mathrm{Nis}} \stackrel{\sim}{\longleftarrow} \beta^{*} R \beta_{*} R j_{*} \mathbb{Z}(n)_{\mathrm{Nis}} \stackrel{\sim}{\longrightarrow} R j_{*} \mathbb{Z}(n)_{\mathrm{Nis}} .
$$


Now the triangle (3) becomes the upper row of the following diagram, which we compare to $R \beta_{*}$ applied to (3) via the adjoint map:

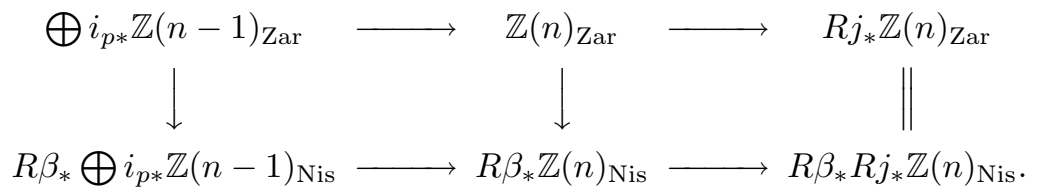

Since $R \beta_{*}$ commutes with pseudo-filtered colimits, the outer maps, hence the middle map is quasi-isomorphism.

\section{Gersten Resolution}

We combine the methods of Bloch [2], [1] and Gillet-Levine [9] to the following more precise version of theorem 1.1 of the introduction:

Theorem 4.1. Let $X \stackrel{p}{\rightarrow} B$ be the local ring at a point $x$ of an essentially smooth scheme over $B$. Let $V$ be the local ring of $X$ at the generic point of the fiber $X_{b}$ over $b=p(x) \in B$. If the map $H^{t}(V, \mathbb{Z}(n)) \rightarrow H^{t}(k(X), \mathbb{Z}(n))$ is injective, then there is an exact sequence

$$
0 \rightarrow H^{t}(X, \mathbb{Z}(n)) \rightarrow \bigoplus_{x \in X^{(0)}} H^{t}(k(x), \mathbb{Z}(n)) \rightarrow \bigoplus_{x \in X^{(1)}} H^{t-1}(k(x), \mathbb{Z}(n-1)) \rightarrow \ldots
$$

Proof. Consider the descending filtration by coniveau on cycles. Let

$$
F^{s} z^{n}(X, *)=\operatorname{colim}_{Z \subseteq X, \operatorname{codim}_{X} Z=s} \operatorname{im}\left(z^{n-s}(Z, *) \rightarrow z^{n}(X, *)\right),
$$

be the subcomplex of $z^{n}(X, *)$ generated by cycles such that the projection to $X$ has codimension at least $s$. The corresponding spectral sequence reads

$$
E_{1}^{s, t}=H_{-s-t}\left(\mathrm{gr}^{s} z^{n}(X, *)\right) \Rightarrow H_{-s-t}\left(z^{n}(X, *)\right)=H^{2 n+s+t}(X, \mathbb{Z}(n)) .
$$

Taking the limit over sequences (4), we get an isomorphism

$$
g r^{s} z^{n}(X, *) \stackrel{\sim}{\longrightarrow} \operatorname{colim}_{(Z, Y)} z^{n-s}(Z-Y, *) \stackrel{r e s}{\sim} \bigoplus_{x \in X^{(s)}} z^{n-s}(k(x), *) .
$$

Here the middle limit runs through all pairs $Y \subseteq Z$ with $Z$ of codimension $s$ and $Y$ of codimension $s+1$, and the right hand map sends a cycle on a subscheme to its restriction to the generic points. Consequently,

$$
E_{1}^{s, t}=H_{-s-t}\left(\mathrm{gr}^{s} z^{n}(X, *)\right)=\bigoplus_{x \in X^{(s)}} H_{-s-t}\left(z^{n-s}(k(x), *)\right)=H^{2 n-s+t}(k(x), \mathbb{Z}(n-s)) .
$$

The sequence of the theorem from the second term on is the sequence of $E_{1}$-terms and differentials. If we can show that the map

$$
H_{m}\left(F^{s+1} z^{n}(X, *)\right) \rightarrow H_{m}\left(F^{s} z^{n}(X, *)\right)
$$

are zero, then the short exact sequences

$$
0 \rightarrow H_{m}\left(F^{s} z^{n}(X, *)\right) \rightarrow H_{m}\left(\mathrm{gr}^{s} z^{n}(X, *)\right) \rightarrow H_{m-1}\left(F^{s+1} z^{n}(X, *)\right) \rightarrow 0
$$

splice together to show that the sequence of $d_{1}$-differentials is exact, except at the first spot $s=0$, where the kernel is $H_{-t}\left(F^{0} z^{n}(X, *)\right)=H^{2 n+t}(X, \mathbb{Z}(n))$.

If $s>0$, then any fixed element $c \in F^{s+1} z^{n}(X, *)$ lies in the image of $F^{s} z^{n-1}(Y, *)$, for $Y$ a principal effective divisor, flat over $\Lambda$. Since the latter has zero boundary 
if the former has zero boundary, the same holds for cohomology classes. Since $X$ is the localization of $X^{\prime}=\operatorname{Spec} S$ in $x$, for $S$ a finitely generated algebra over the discrete valuation ring $\Lambda=B_{b}$, we can (after decreasing $X^{\prime}$ ) assume that $c$ is the restriction of an element in $F^{s+1} z^{n}\left(X^{\prime}, *\right)$ and comes from $Y^{\prime}=\operatorname{Spec} S / t S$, where $t$ is a regular element of $S$. We can always replace Spec $S$ by a smaller affine neighborhood of $x$.

By [9, Lemma 1, Section 2], after replacing $X^{\prime}$ by a smaller affine neighborhood of $x$, we can find a morphism $\pi: X^{\prime} \rightarrow \mathbb{A}_{\Lambda}^{d-1}$, such that $\pi$ is smooth at $x$ of relative dimension 1 , and $\left.\pi\right|_{Y^{\prime}}$ is quasi-finite. Setting $T=S / t \otimes_{\mathbb{A}_{\Lambda}^{d-1}} S$ and $Z=\operatorname{Spec} T=$ $Y^{\prime} \times_{\mathbb{A}_{\Lambda}^{d-1}} X^{\prime}$, we get the cartesian square

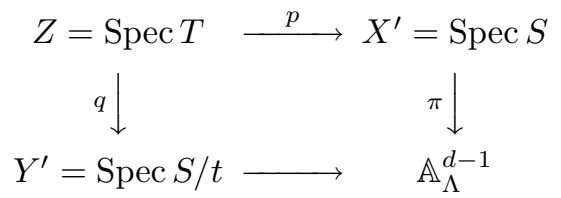

The inclusion $Y^{\prime} \stackrel{j}{\rightarrow} X^{\prime}$ induces a section $s: Y^{\prime} \rightarrow Z$ of $q$, which is a closed immersion. Its image $s\left(Y^{\prime}\right)$ is finite over $X^{\prime}$. Furthermore $q$ is smooth at the points $p^{-1}(x)$, and $p$ is quasi-finite. By Zariski's main theorem (EGA IV, 18.12.13) there is an affine scheme finite over $X^{\prime}, \bar{Z}=\operatorname{Spec} \bar{T} \stackrel{\bar{p}}{\rightarrow} X^{\prime}$, with $Z \stackrel{u}{\longrightarrow} \bar{Z}$ an open immersion. Let $\bar{s}=u \circ s: Y^{\prime} \rightarrow \bar{Z}$. Since $j=\bar{p} \circ \bar{s}$ is closed, $\bar{s}$ is closed and $D=\bar{Z}-Z$ is disjoint from $\bar{s}\left(Y^{\prime}\right)$. Since $\bar{p}$ is proper, we can factor $j_{*}=\bar{p}_{*} \bar{s}_{*}$, and it suffices to show that $\bar{s}_{*}=0$.

Since $Z$ is smooth over $Y^{\prime}$ of relative dimension 1 at the finite set of points of $p^{-1}(x)$, the ideal $I \subset T$ defining $s\left(Y^{\prime}\right)$ is principal near these points (SGA1, II 4.15), say $I=\left(g^{\prime}\right)$. Let $\bar{I} \subset \bar{T}$ be the ideal defining $\bar{s}(Y)$, and $J \subset \bar{T}$ the ideal defining $D$. Because $\bar{s}\left(Y^{\prime}\right) \cap D=\emptyset$, we can use the Chinese remainder theorem to find $g \in \bar{T}$ which maps to $\left(1, g^{\prime}\right) \in \bar{T} / J \oplus \bar{T} / \bar{I}^{2}$, where $g^{\prime}$ is viewed as an element of $\bar{T} / \bar{I}^{2}$ via the isomorphism $\bar{T} / \bar{I}^{2} \stackrel{\sim}{\longrightarrow} T / I^{2}$. Then $g=1$ on $D$ and $g$ defines $\bar{s}(Y)$ in a neighborhood of $\bar{p}^{-1}(x)$. Let $C$ be the closed set of $\bar{Z}$ consisting of all irreducible components of the divisor $g=0$ which are not components of $\bar{s}\left(Y^{\prime}\right)$. Then $C$ does not contain any point of $\bar{p}^{-1}(x)$, so its image $\bar{p}(C) \in X^{\prime}$ is a closed subset not containing $x$. By removing $\bar{p}(C)$ from $X^{\prime}$, we can assume that the divisor $g=0$ is $\bar{s}\left(Y^{\prime}\right)$.

Let $y \in z^{n-1}\left(Y^{\prime}, t\right), z=q^{*} y \in z^{n-1}(Z, t)$ and $\bar{z}$ be the closure of $z$ in $\bar{Z} \times \Delta^{t}$. Consider the closed embeddings

$$
\begin{aligned}
& \gamma=\left(\text { id, } \frac{g}{g-1}\right): Z \rightarrow Z \times \mathbb{P}^{1}, \\
& \bar{\gamma}=\left(\text { id, } \frac{g}{g-1}\right): \bar{Z} \rightarrow \bar{Z} \times \mathbb{P}^{1},
\end{aligned}
$$

and let $\gamma_{*}: z^{n-1}(Z, t) \rightarrow z^{n}\left(Z \times \mathbb{P}^{1}, t\right) \stackrel{\text { res }}{\longrightarrow} z^{n}\left(Z \times \mathbb{A}^{1}, t\right)$ be the induced map on cycles. Since $\frac{g}{g-1}$ has a pole on $D, \gamma_{*}(z)$ agrees with the restriction of $\bar{\gamma}_{*}(\bar{z})$ to $\bar{Z} \times \mathbb{A}^{1} \times \Delta^{t}$, hence is closed in $\bar{Z} \times \mathbb{A}^{1} \times \Delta^{t}$ and can be viewed as an element of $z^{n}\left(\bar{Z} \times \mathbb{A}^{1}, t\right)$. By homotopy invariance, the two maps $i_{0}^{*}, i_{1}^{*}$ induced by the points $0,1 \in \mathbb{A}^{1}$ induce homotopic maps $z^{n}\left(\bar{Z} \times \mathbb{A}^{1}, *\right) \rightarrow z^{n}(\bar{Z}, *)$. Since $g$ has no poles, the image of $\bar{\gamma}_{*}$ does not meet $\bar{Z} \times\{1\}$, and $i_{1}^{*}$ is the zero map. Consider the 
cartesian square

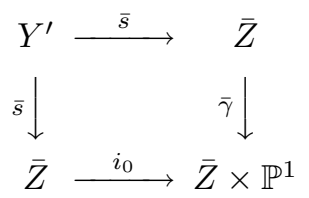

Since $\bar{z}$ is a cycle on $\bar{Z}$ which meets $Y^{\prime}$ properly because of $\bar{z} \cap \bar{s}\left(Y^{\prime}\right)=q^{*} y \cap s\left(Y^{\prime}\right)$, the base change for algebraic cycles gives $i_{0}^{*} \bar{\gamma}_{*} \bar{z}=\bar{s}_{*} \bar{s}^{*} \bar{z}$. Because the divisor $\bar{s}\left(Y^{\prime}\right)$ defined by $g=0$ is disjoint from $D$, we have

$$
i_{0}^{*} \gamma_{*}(z)=i_{0}^{*} \bar{\gamma}_{*}(\bar{z})=\bar{s}_{*} \bar{s}^{*}(\bar{z})=\bar{s}_{*} s^{*}(z)=\bar{s}_{*} s^{*} q^{*}(y)=\bar{s}_{*}(y)
$$

This shows that the inclusion $F^{s+1} z^{n}(X, *) \hookrightarrow F^{s} z^{n}(X, *)$ induces the zero map on homology. The analog statement for $s=0$ is by (4) equivalent to the injectivity of the map $H^{t}(X, \mathbb{Z}(n)) \rightarrow H^{t}(k(X), \mathbb{Z}(n))$. By the same argument as above, $H^{t}(X, \mathbb{Z}(n)) \rightarrow H^{t}(, V \mathbb{Z}(n))$ is injective, because Spec $V$ arises from $X$ by removing flat divisors. On the other hand, the map $H^{t}(V, \mathbb{Z}(n)) \rightarrow H^{t}(k(X), \mathbb{Z}(n))$ is injective by hypothesis.

Corollary 4.2. Let $X$ be an essentially smooth scheme over $B$. Then $\mathcal{H}^{i}(\mathbb{Z}(n))=0$ for $i>n$.

Proof. We can assume that $X$ is local. Let $R$ be the local ring of a generic point of the closed fiber of $X, k$ the residue field and $K$ the field of quotients of $R$. We have shown in the proof of theorem 4.1 that $H^{i}(X, \mathbb{Z}(n))$ injects into $H^{i}(R, \mathbb{Z}(n))$. From the localization sequence

$$
\cdots \rightarrow H^{s}(R, \mathbb{Z}(n)) \rightarrow H^{s}(K, \mathbb{Z}(n)) \stackrel{\delta}{\rightarrow} H^{s-1}(k, \mathbb{Z}(n-1)) \rightarrow \cdots,
$$

we get $H^{s}(R, \mathbb{Z}(n))=0$ for $s>n+1$. On the other hand, $H^{n+1}(R, \mathbb{Z}(n))=0$ because the symbol map $K_{n}^{M}(K) \rightarrow K_{n-1}^{M}(k)$ is surjective.

Corollary 4.3. Let $X$ be a smooth scheme over a discrete valuation ring with residue characteristic $p$. Then there is an exact sequence

$$
\begin{aligned}
0 \rightarrow \mathcal{H}^{s}\left(\mathbb{Z} / p^{r}(n)_{\text {Zar }}\right) & \rightarrow \bigoplus_{x \in X^{(0)}}\left(i_{x}\right)_{*} H^{s}\left(k(x), \mathbb{Z} / p^{r}(n)\right) \\
& \rightarrow \bigoplus_{x \in X^{(1)}}\left(i_{x}\right)_{*} H^{s-1}\left(k(x), \mathbb{Z} / p^{r}(n-1)\right) \rightarrow \cdots
\end{aligned}
$$

Proof. Let $R$ be the local ring of a generic point of the closed fiber of $X, k$ the residue field and $K$ the field of quotients of $R$. We have to show that in the localization sequence

$$
\cdots \rightarrow H^{s}\left(R, \mathbb{Z} / p^{r}(n)\right) \rightarrow H^{s}\left(K, \mathbb{Z} / p^{r}(n)\right) \stackrel{\delta}{\rightarrow} H^{s-1}\left(k, \mathbb{Z} / p^{r}(n-1)\right) \rightarrow \cdots
$$

the map $\delta$ is surjective. By [7] the right group is trivial for $s \neq n$, and agrees with $K_{n-1}^{M}(k) / p^{r}$ for $s=n$. Let $t$ be a uniformizer of $R$. Then we can lift any symbol $\left\{x_{1}, \ldots, x_{n-1}\right\}$ of the latter group to the symbol

$$
\left\{x_{1}, \ldots, x_{n-1}, t\right\} \in K_{n}^{M}(K) / p^{r} \cong H^{n}\left(K, \mathbb{Z} / p^{r}(n)\right) .
$$




\section{Etale motivic COHOMology}

In this section we study étale motivic cohomology. Let us first discuss rational motivic cohomology:

Proposition 5.1. If $X$ be an essentially smooth scheme over $B$, then

$$
\mathbb{Q}(n)_{\mathrm{Zar}} \cong R \epsilon_{*} \mathbb{Q}(n)_{\text {ét. }} .
$$

Proof. We first prove the theorem for $X$ a smooth variety over a field. By replacing $X$ by an étale scheme $V$ over $X$ it suffices to show $H_{\text {Zar }}^{s}(X, \mathbb{Q}(n)) \stackrel{\sim}{\longrightarrow} H_{\text {ét }}^{s}(X, \mathbb{Q}(n))$. For a point $x \in X$ let $H_{x}^{s, n}$ be the presheaf on $E t / k(x)$ which maps $k^{\prime} / k(x)$ to $H_{\text {ét }}^{s}\left(k^{\prime}, \mathbb{Q}(n)\right)$. Since higher cohomology of a profinite group is torsion, the spectral sequence for the cohomology of a complex shows that this presheaf agrees with $z^{n}(-, 2 n-s) \otimes \mathbb{Q}$, which is a sheaf by lemma 3.1. Now if $U$ is étale over $X$, then

$$
\left(i_{x}\right)_{*} H_{x}^{s, n}(U)=H_{\text {êt }}^{s}\left(U \times_{X} k(x), \mathbb{Q}(n)\right) \cong \bigoplus_{U \ni u \mapsto x} H_{\text {ét }}^{s}(k(u), \mathbb{Q}(n)) .
$$

Thus the sheafification of the presheaf of exact sequences coming from the Gersten resolution gives the following exact sequence of étale sheaves

$$
0 \rightarrow \mathcal{H}^{s}\left(\mathbb{Q}(n)_{\text {ét }}\right) \rightarrow \bigoplus_{x \in X^{(0)}}\left(i_{x}\right)_{*} H_{x}^{s, n} \rightarrow \bigoplus_{x \in X^{(1)}}\left(i_{x}\right)_{*} H_{x}^{s-1, n-1} \rightarrow \cdots
$$

Since higher cohomology of a profinite group is torsion, we get for any $x \in X$

$$
H_{\text {ét }}^{t}\left(X,\left(i_{x}\right)_{*} H_{x}^{s, n}\right)=H_{\text {ét }}^{t}\left(k(x), H_{x}^{s, n}\right)= \begin{cases}H_{\text {ét }}^{s}(k(x), \mathbb{Q}(n)) & t=0 \\ 0 & t>0 .\end{cases}
$$

Hence the terms in (9) are acyclic for $\Gamma_{\text {ét }}(X,-)$, and we can calculate étale rational motivic cohomology with this resolution. On the other hand,

$$
\begin{aligned}
& H_{\mathrm{Zar}}^{s}(k(x), \mathbb{Q}(n)) \stackrel{\sim}{\longrightarrow} H_{\mathrm{Zar}}^{s}\left(k(x)^{\mathrm{sep}}, \mathbb{Q}(n)\right)^{\operatorname{Gal}(k(x))} \\
& \quad=H_{\text {êt }}^{s}\left(k(x)^{\operatorname{sep}}, \mathbb{Q}(n)\right)^{\operatorname{Gal}(k(x))} \stackrel{\sim}{\sim} H_{\text {êt }}^{s}(k(x), \mathbb{Q}(n)) .
\end{aligned}
$$

The first equality follows using trace maps, and the third equality comes from the degeneration of the Hochschild-Serre spectral sequence. Thus applying $\Gamma_{\text {ét }}(X,-)$ to $(9)$ and $\Gamma_{\mathrm{Zar}}(X,-)$ to the Gersten resolution gives isomorphic sequences, hence an isomorphism $H_{\text {Zar }}^{s}(X, \mathbb{Q}(n)) \stackrel{\sim}{\longrightarrow} H_{\text {ét }}^{s}(X, \mathbb{Q}(n))$.

To generalize the theorem from a field as a base to a Dedekind ring as a base, we repeat the proof of proposition 3.6, replacing $\mathbb{Z}(n), N i s$, and $\beta$ by $\mathbb{Q}(n)$, ét, and $\epsilon$, respectively.

Our next goal is to understand the étale motivic cohomology sheaves with finite coefficients. Over a field of characteristic $p$, one has $[7,8]$

$$
\mathbb{Z} / m(n)_{\text {ét }} \cong \mu_{m}^{\otimes n} \text { for } p \nmid m_{m}, \quad \mathbb{Z} / p^{r}(n)_{\text {ét }} \cong \nu_{r}^{n}[-n] .
$$

Here $\nu_{r}^{n}=\nu_{p^{r}}(n)=W \Omega_{r, l o g}^{n}$ is the logarithmic de Rham-Witt sheaf. One expects the following weaker version of localization:

Conjecture 5.2. (Purity) Let $X$ be essentially smooth over $B$, and $i: X_{b} \rightarrow X$ be the inclusion of the closed fiber over $b \in B$. Then there is a quasi-isomorphism

$$
\mathbb{Z}(n-1)_{\text {ét }}[-2] \stackrel{\sim}{\longrightarrow} \tau_{\leq n+1} R i ! \mathbb{Z}(n)_{\text {ét }} .
$$


Moreover, truncation is only necessary for $n=0$ and for the mod $p$-part, $p$ the characteristic of $k(b)$.

We now prove theorem 1.2 of the introduction.

Proof. 1) In fact we prove the stronger statement of conjecture 5.2. The proof is a variant of the proof of proposition 3.6, keeping track of the range of validity. Both conjecture 5.2 and the Beilinson-Lichtenbaum conjecture hold with rational coefficient without truncation by proposition 5.1. To show that the Bloch-Kato conjecture implies conjecture 5.2, we can assume as in proposition 3.6 that $B$ is local. Applying $\epsilon^{*} i^{*}$ to (6) and comparing to (3) via the adjoint map, we get the diagram

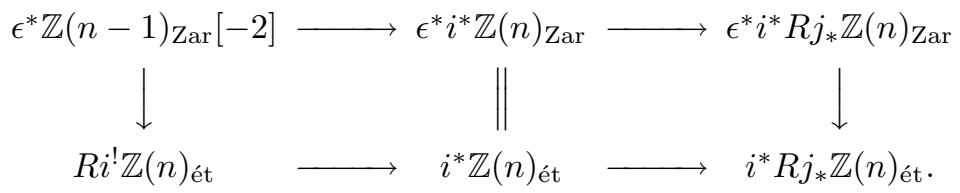

The right hand map is quasi-isomorphism up to degree $n+1$, because the BeilinsonLichtenbaum conjecture over a field implies that

$$
\epsilon^{*} R j_{*} \mathbb{Z}(n)_{\mathrm{Zar}} \stackrel{\sim}{\longrightarrow} \tau_{\leq n+1} \epsilon^{*} R j_{*} R \epsilon_{*} \mathbb{Z}(n)_{\text {ét }} \stackrel{\sim}{\longrightarrow} \tau_{\leq n+1} R j_{*} \mathbb{Z}(n)_{\text {ét }} .
$$

Thus the left map is a quasi-isomorphism up to degree $n+1$. Now $\mathbb{Z}(n-1)$ ét $[-2]$ is acyclic above degree $n+1$ by corollary 4.2. If $m$ is invertible on the fiber over $b$ and $n>0$, then the right two terms of the lower sequence of (5) are acyclic with $\mathbb{Z} / m$-coefficients above degree $n$ in view of absolute cohomological purity:

$$
R^{s} j_{*} \mathbb{Z} / m(n)_{\text {ét }} \cong R^{s} j_{*} \mu_{m}^{\otimes n} \cong \begin{cases}\mu_{m}^{\otimes n} & s=0 \\ \mu_{m}^{\otimes n-1} & s=1 \\ 0 & \text { otherwise. }\end{cases}
$$

Hence $R i^{!} \mathbb{Z} / m(n)_{\text {ét }}$ is acyclic above degree $n+1$, and truncation is not necessary.

2) The Bloch-Kato conjecture implies the Beilinson-Lichtenbaum conjecture over a field, see $[7,8]$. In general, we can consider stalks and assume that the base is local. We compare the triangle (6) to $R \epsilon_{*}$ applied to (3) via the adjoint map:

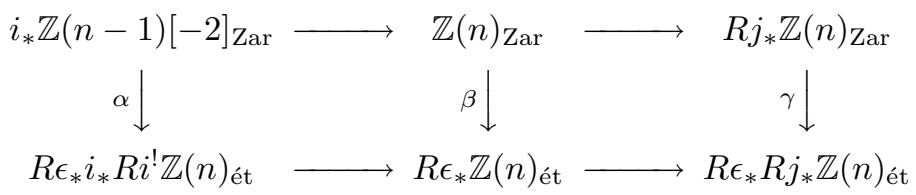

The maps $\alpha$ and $\gamma$ is a quasi-isomorphism up to degree $n+1$, because the conjecture holds over fields and because

$$
i_{*} \mathbb{Z}(n-1)_{\text {Zar }}[-2] \stackrel{\sim}{\sim} \tau_{\leq n+1} i_{*} R \epsilon_{*} \mathbb{Z}(n-1)_{\text {ét }}[-2] \stackrel{\sim}{\longrightarrow} \tau_{\leq n+1} i_{*} R \epsilon_{*} R i ! \mathbb{Z}(n)_{\text {ét }} .
$$

On the other hand, $\alpha$ is injective in degree $n+2$ because the domain is 0 . By the five-lemma, $\beta$ is a quasi-isomorphism up to degree $n+1$.

3) To prove rigidity, consider the diagram

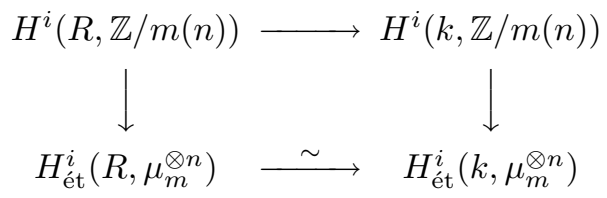


The lower horizontal arrow is an isomorphism by rigitiy for étale cohomology, and the vertical maps are isomorphisms for $i \leq n+1$ by the Beilinson-Lichtenbaum conjecture. On the other hand, the upper terms are both zero for $i>n$ by corollary 4.2 .

4) Compare the following two distinguished triangles of (3) via the cycle class $\operatorname{map} \mathbb{Z} / m(n)_{\text {ét }} \stackrel{c l_{X}^{n}}{\longrightarrow} \mu_{m}^{\otimes n}$ of [16, par. 12.3] or [8]:

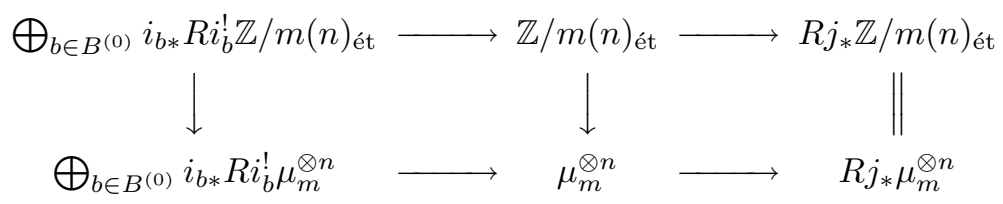

The right hand map is an isomorphism by (10). By part (1) and purity for $\mu_{m}^{\otimes n}$, we get $R i_{b}^{!} \mathbb{Z} / m(n)_{\text {ét }} \cong \mathbb{Z} / m(n-1)_{\text {ét }}[-2] \cong \mu_{m}^{\otimes n-1}[-2] \cong R i^{!} \mu_{m}^{\otimes n}$ for every closed fiber, hence the middle map is a quasi-isomorphism.

5) It suffices to check this for a local ring $R$ of $X$ and for $m$ a power of some prime number $l$. If $R$ has residue characteristic $l$, then the statement was proved in corollary 4.3. If $l$ is invertible in $R$, then by theorem $4.1,(2)$ and (4), it suffices to show that the map $H_{\text {êt }}^{s}\left(R, \mu_{m}^{\otimes n}\right) \rightarrow H_{\text {ét }}^{s}\left(k(R), \mu_{m}^{\otimes n}\right)$ is injective. This is $[4$, theorem B.2.1].

Remark. Using the product with equidimensional cycles defined by Levine [16], one can show under the same hypothesis that $\mu_{m}^{\otimes n}$ is a split direct summand of $\mathbb{Z} / m(n)_{\text {ét. }}$ This is because the cycle class map is split by the composition

$$
\mu_{m}^{\otimes n} \underset{\sim}{\stackrel{c l 1}{\stackrel{1}{\sim}}} \mathbb{Z} / m(1)^{\otimes n} \stackrel{\cup}{\longrightarrow} \mathbb{Z} / m(n) .
$$

The cup product is defined because we are multiplying with one-codimensional cycles, and the composition with the cycle class map is the identity because the cycle class map is multiplicative [8, Prop. 4.7].

We define the $p$-cohomological dimension $\mathrm{cd}_{p} X_{\text {ét }}$ of the site $X_{\text {ét }}$ to be the smallest integer $n$ such that $H_{\text {ét }}^{i}(V, F)=0$ for all $i>n$, any scheme $V$ étale over $X$ and any $p$-torsion sheaf $F$.

Proposition 5.3. Let $X$ be essentially smooth over $B$ with a closed fiber $i: X_{b} \rightarrow$ $X$ of residue characteristic $p$ and generic fiber $U$. Then conjecture 5.2 implies

$$
R i^{\prime} \mathbb{Z}(n)_{\text {ét }} \cong \mathbb{Z}(n-1)_{\text {ét }}[-2] \quad \text { for } n \geq \operatorname{cd}_{p} U_{\text {ét }} .
$$

Proof. In view of proposition 5.1, corollary 3.3 and theorem 1.2(4), the sheaves $R^{s} i^{!} \mathbb{Z}(n)$ ét are torsion and $m$-divisible for $p \wedge m$ and $s>n+1$. By a diagram chase it suffices to show that $R^{s} i^{!} \mathbb{Z} / p(n)_{\text {ét }}=0$ for $s>n+1 \geq \operatorname{cd}_{p} U+1$. By excision we can furthermore assume that $B$ is local. As $\mathcal{H}^{s}\left(\mathbb{Z} / p(n)_{\text {ét }}\right)=0$ for $s>n$, the long exact decomposition sequence gives isomorphisms $i^{*} R^{s-1} j_{*} \mu_{p}^{\otimes n} \stackrel{\sim}{\longrightarrow}$ $R^{s} i ! \mathbb{Z} / p(n)_{\text {ét }}$, for $s>n+1$. The stalk of the left hand side at a point $y$ of $X_{b}$ is $H_{\text {ét }}^{s-1}\left(\operatorname{Spec} \mathcal{O}_{X, y}^{s h} \times_{X} U, \mu_{p}^{\otimes n}\right)$, and this vanishes for $s>\operatorname{cd}_{p} U+1$ because étale cohomology commutes with inverse limits of affine schemes. 


\section{Motivic COHOMOLOGY AND SYNTOMic COHOMOLOGY}

In this section we restrict ourselves to the case that $B$ is the spectrum of a discrete valuation ring $V$ of characteristic $(0, p)$ and uniformizer $\pi$. If $i: Y \rightarrow X$ is the embedding of the closed fiber, then conjecture 5.2 with $\mathbb{Z} / p^{r}$-coefficients takes the form

$$
R^{s} i^{!} \mathbb{Z} / p^{r}(n)_{\text {ét }} \cong \begin{cases}0 & s \leq n \\ \nu_{r}^{n-1} & s=n+1 .\end{cases}
$$

Let $S_{r}(n)$ be the syntomic complex of Fontaine-Messing [6], a complex of étale sheaves on $Y$. By a result of Kato and Kurihara [13], there is an exact triangle for the étale syntomic sheaves $S_{r}(n)$, at least for $n<p-1$,

$$
\cdots \rightarrow S_{r}(n) \rightarrow \tau_{\leq n} i^{*} R j_{*} \mu_{p^{r}}^{\otimes n} \stackrel{\kappa}{\longrightarrow} \nu_{r}^{n-1}[-n] \rightarrow \cdots
$$

The map $\kappa$ is the composition of the canonical map $\tau_{\leq n} i^{*} R j_{*} \mu_{p^{r}}^{\otimes n} \rightarrow i^{*} R^{n} j_{*} \mu_{p^{r}}^{\otimes n}[-n]$ with the symbol map of [3, par. 6.6]. More precisely, $i^{*} R^{n} j_{*} \mu_{p^{r}}^{\otimes n}[-n]$ is locally generated by symbols $\left\{f_{1}, \ldots, f_{n}\right\}$, for $f_{i} \in i^{*} j_{*} \mathcal{O}_{U}^{\times}$by $[3$, cor. 6.1.1]. By multilinearity, each such symbol can be written as a sum of symbols of the form $\left\{f_{1}, \ldots, f_{n}\right\}$ and $\left\{f_{1}, \ldots, f_{n-1}, \pi\right\}$, for $f_{i} \in i^{*} \mathcal{O}_{X}^{\times}$. Then $\kappa$ sends the former to zero, and the latter to $\left\{d \log \bar{f}_{1}, \ldots, d \log \bar{f}_{n-1}\right\}$, where $\bar{f}_{i}$ is the reduction of $f_{i}$ to $\mathcal{O}_{Y}^{\times}$. For $n \geq p-1$, we can define $S_{r}(n)$ to be the cone of the map $\kappa$. This cone has been studied by Sato [22]. We now prove theorem 1.3 of the introduction:

Proof. In view of corollary 4.3 , we have $\mathcal{H}^{s}\left(\mathbb{Z} / p^{r}(n)\right)=0$ for $s>n$, so that we can replace the motivic complex by its truncation $\tau_{\leq n} \mathbb{Z} / p^{r}(n)$ Zar. We then have the following diagram of exact triangles in $D\left(S h v_{\text {ét }}(\bar{Y})\right)$ :

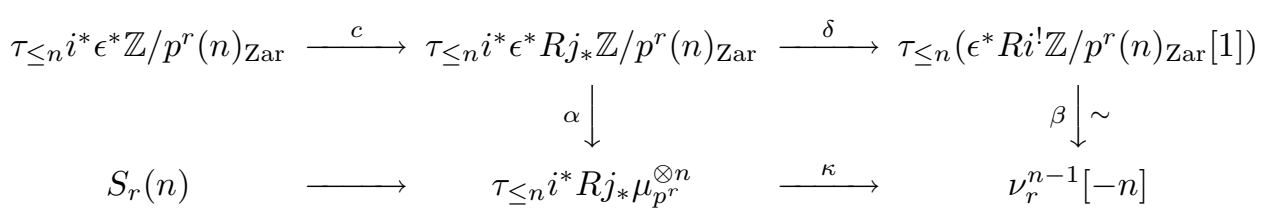

Here $\alpha$ is the composition of the adjoint map $\epsilon^{*} R j_{*} \rightarrow R j_{*} \epsilon^{*}$ and the cycle map $\mathbb{Z} / p^{r}(n)_{\text {ét }} \rightarrow \mu_{p^{r}}^{\otimes n}$ of [8], and $\beta$ is the quasi-isomorphism

$$
\tau_{\leq n}\left(\epsilon^{*} R i^{!} \mathbb{Z} / p^{r}(n)_{\operatorname{Zar}}[1]\right) \stackrel{\sim}{\sim} \tau_{\leq n}\left(\epsilon^{*} \mathbb{Z} / p^{r}(n-1)_{\operatorname{Zar}}[-1]\right) \stackrel{\sim}{\longrightarrow} \nu_{r}^{n-1}[-n] .
$$

Since $\operatorname{Hom}\left(\tau_{\leq n} i^{*} \epsilon^{*} \mathbb{Z} / p^{r}(n)_{\mathrm{Zar}}, \nu_{r}^{n-1}[-n-1]\right)=0$ for degree reasons, we get an exact sequence of groups of homomorphisms in the derived category

$$
\begin{aligned}
& 0 \rightarrow \operatorname{Hom}\left(\tau_{\leq n} i^{*} \epsilon^{*} \mathbb{Z} / p^{r}(n)_{\mathrm{Zar}}, S_{r}(n)\right) \longrightarrow \\
& \operatorname{Hom}\left(\tau_{\leq n} i^{*} \epsilon^{*} \mathbb{Z} / p^{r}(n)_{\mathrm{Zar}}, \tau_{\leq n} i^{*} R j_{*} \mu_{p^{r}}^{\otimes n}\right) \stackrel{\kappa \circ}{\longrightarrow} \operatorname{Hom}\left(\tau_{\leq n} i^{*} \epsilon^{*} \mathbb{Z} / p^{r}(n)_{\mathrm{Zar}}, \nu_{r}^{n-1}[-n]\right)
\end{aligned}
$$

This shows that there can be at most one map making the diagram commute, and that we can lift $\alpha \circ c$ to the desired map if $\kappa \circ(\alpha \circ c)=0$. Again for degree reasons, the last group injects into $\operatorname{Hom}\left(\mathcal{H}^{n}\left(i^{*} \epsilon^{*} \mathbb{Z} / p^{r}(n)_{\mathrm{Zar}}\right), \nu_{r}^{n-1}[-n]\right)$, and we only have to check that $\kappa \circ \alpha \circ c=0$ in degree $n$. Thus it suffices to show that for a strictly Henselian local ring $R$ of $X$ in a point of the closed fiber, the composition

$$
H^{n}\left(R, \mathbb{Z} / p^{r}(n)\right) \stackrel{c}{\longrightarrow} H^{n}\left(R\left[\frac{1}{\pi}\right], \mathbb{Z} / p^{r}(n)\right) \stackrel{\alpha}{\longrightarrow} H_{\text {ét }}^{n}\left(R\left[\frac{1}{\pi}\right], \mu_{p^{r}}^{\otimes n}\right) \stackrel{\kappa}{\longrightarrow} \nu_{r}^{n-1}(R / \pi)
$$

is the zero map. Let $L$ be the field of quotients of $R$, and $F$ be the field of quotients of $R / \pi$. The local ring $D$ of the generic point of the closed fiber $\operatorname{Spec} R / \pi$ is a discrete valuation ring with quotient field $L$ and residue field $F$. Let $D^{h}$ be 
the Henselization of $D, L^{h}$ be the quotient field of $D$, and consider the following commutative diagram, where the vertical maps different from $c$ are induced by inclusions of rings:

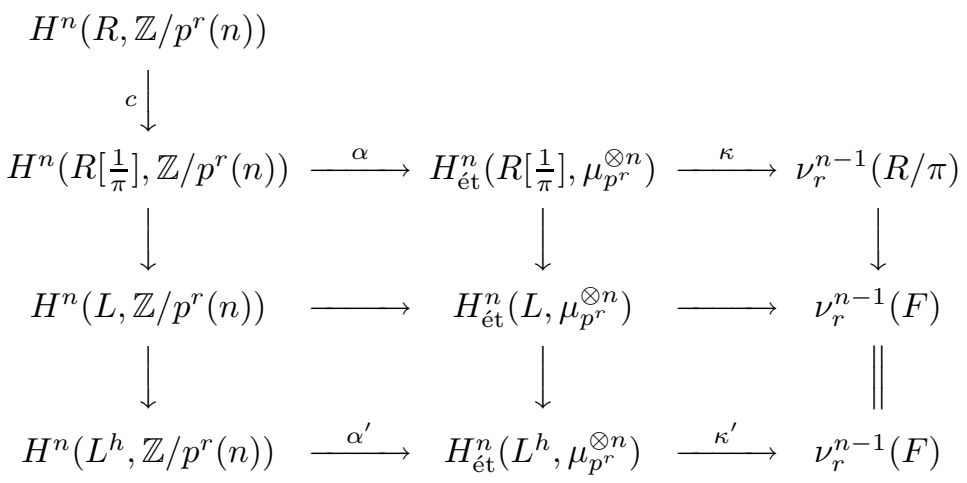

Since the right vertical map is injective by Gros-Suwa [10], it suffices to show that the total composition is the zero map. The composition

$H^{n}\left(R, \mathbb{Z} / p^{r}(n)\right) \rightarrow H^{n}\left(D^{h}, \mathbb{Z} / p^{r}(n)\right) \rightarrow H^{n}\left(L^{h}, \mathbb{Z} / p^{r}(n)\right) \stackrel{\delta}{\rightarrow} H^{n-1}\left(F, \mathbb{Z} / p^{r}(n-1)\right)$,

where the last two maps are the maps of the localization sequence, is zero. Thus is suffices to show that the right square in following diagram commutes:

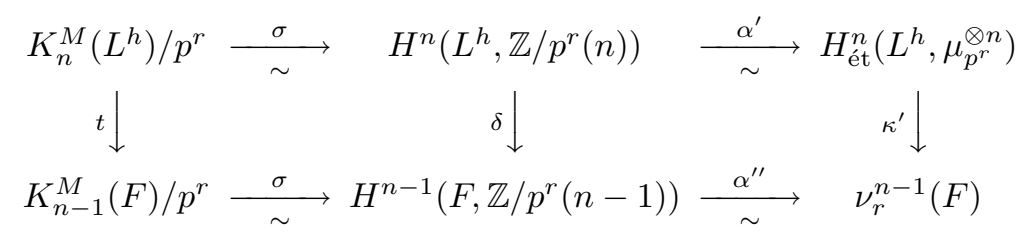

Here $t$ is the tame symbol and $\sigma$ is the map of Nesterenkov and Suslin, which is multiplicative by [7, prop. 3.3]. On the other hand, $\alpha^{\prime}$ is multiplicative by $[8$, prop. 4.7]. In particular, $\alpha^{\prime} \circ \sigma$ is the symbol map to étale cohomology, and the outer square commutes by the definition of $\kappa^{\prime}$. The left square commutes by the argument of [7, lemma 3.2], hence the right square commutes.

Clearly, the map $i^{*} \mathbb{Z} / p^{r}(n)_{\text {ét }} \rightarrow S_{r}(n)$ is a quasi-isomorphism if and only if $\alpha$ is a quasi-isomorphism. Comparing the étale sheafification of Zariski motivic cohomology to étale motivic cohomology

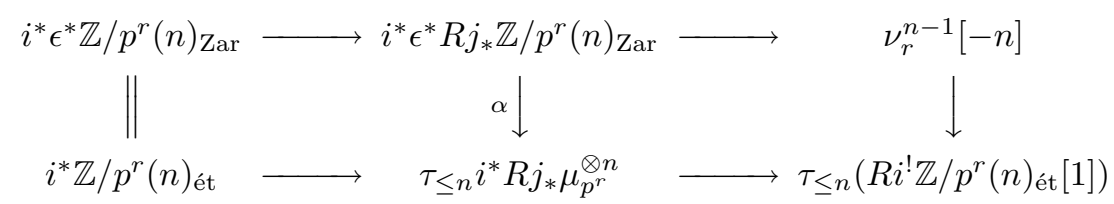

we see that $\alpha$ is a quasi-isomorphism if conjecture 5.2 holds.

Remark. Again one can show that $\nu_{r}^{n-1}[-n-1]$ is a split direct summand of $\tau_{\leq n+1} R i^{!} \mathbb{Z} / p^{r}(n)_{\text {ét. }}$. The inverse map (in the derived category) to the canonical 
map comes through the commutative diagram

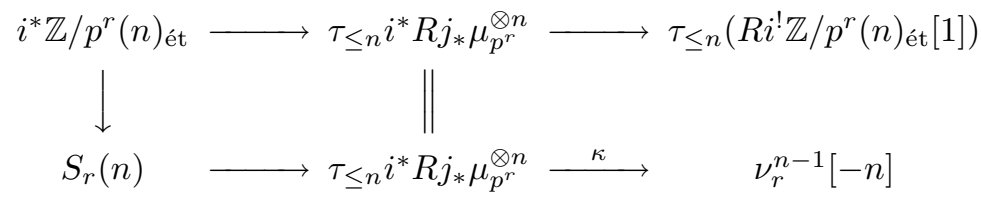

There is exactly one map $s$ mapping to the map $\kappa$ in the third group in the following exact sequence:

$$
\begin{aligned}
& 0 \rightarrow \operatorname{Hom}\left(\tau_{\leq n}\left(R i^{!} \mathbb{Z} / p^{r}(n)_{\text {ét }}[1], \nu_{r}^{n-1}[-n]\right) \rightarrow\right. \\
& \operatorname{Hom}\left(\tau_{\leq n} i^{*} R j_{*} \mu_{p^{r}}^{\otimes n}, \nu_{r}^{n-1}[-n]\right) \rightarrow \operatorname{Hom}\left(i^{*} \mathbb{Z} / p^{r}(n)_{\text {ét }}, \nu_{r}^{n-1}[-n]\right)
\end{aligned}
$$

It is easy to verify that $s$ is a splitting of the natural map.

Remark. The above shows the relationship of the different proofs of the crystalline conjecture. Let $\bar{V}$ be the ring of integers of the algebraic closure of a $p$-adic field (note that $\bar{V}$ is not a discrete valuation ring, but it is the direct limit of discrete valuation rings, so our methods still apply). Analog to Niziol [20], we get a commutative diagram for $n<p-1$ and $X$ smooth and proper over $B$,

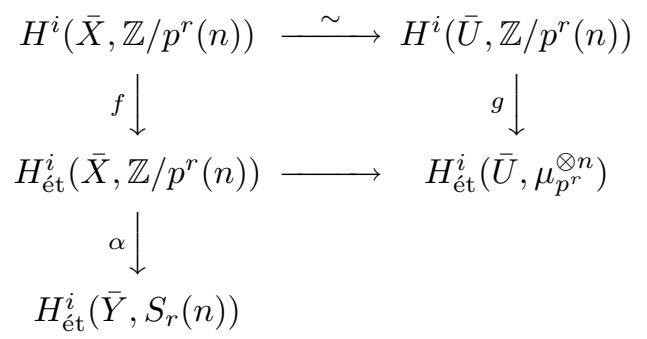

For $\operatorname{dim} U \leq n$, the map $g$ is an isomorphism by Suslin [25]. Thus for $p-2 \geq n \geq$ $\operatorname{dim} U$ we get the map

$$
H_{\text {êt }}^{i}\left(\bar{U}, \mu_{p^{r}}^{\otimes n}\right) \rightarrow H_{\text {êt }}^{i}\left(\bar{Y}, S_{r}(n)\right)
$$

needed to prove the crystalline conjecture. However, our result assumes [13], which is the essential ingredient of Kato's proof of the conjecture.

Remark. Theorem 1.3 gives a cycle map to syntomic cohomology, hence to crystalline cohomology. The adjoint map $\mathbb{Z} / p^{r}(n)_{\text {Zar }} \rightarrow R \epsilon_{*} \mathbb{Z} / p^{r}(n)_{\text {ét }}$ gives rise to a map in degree $2 n$,

$$
\mathrm{CH}^{n}(X) / p^{r} \cong H^{2 n}\left(X, \mathbb{Z} / p^{r}(n)\right) \rightarrow H_{\text {ét }}^{2 n}\left(X, \mathbb{Z} / p^{r}(n)\right)
$$


For $X$ smooth and proper over $B$ this fits into the following diagram (the right column exists for $p>n+1)$ :

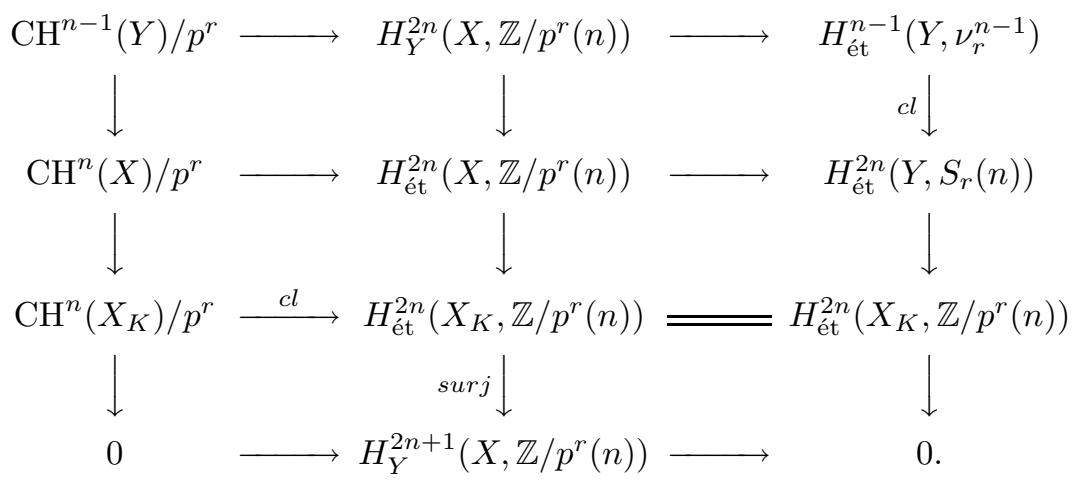

The left upper map factors through $H_{\text {ét }}^{n-1}\left(Y, \mathbb{Z} / p^{r}(n-1)\right)$. Thus étale motivic cohomology is a good recipient of the cycle map (generalizing syntomic cohomology), and we hope that our map can be useful in studying algebraic cycles on smooth schemes over discrete valuation rings.

\section{REFERENCES}

[1] S.Bloch, Algebraic Cycles and Higher $K$-theory, Adv. in Math. 61 (1986), 267-304.

[2] S.Bloch, A note on Gersten's conjecture in the mixed characteristic case, Appl. of Alg. $K$-theory to Alg. Geometry and Number Theory, Cont. Math. 55 Part I (1986), 75-78.

[3] S.Bloch, K.Kato, p-adic étale cohomology, Publ. Math. IHES 63 (1986), 147-164.

[4] J.L. Colliot-Thélène, R.Hoobler, B.Kahn, The Bloch-Ogus-Gabber theorem, Fields Inst. Com. 16 (1997), 31-94.

[5] E.Friedlander, A.Suslin, The spectral sequence relating algebraic K-theory to motivic cohomology, Preprint 1999

[6] J.M.Fontaine, W.Messing, p-adic periods and p-adic étale cohomology, Cont. Math. 67(1987), 179-207.

[7] T.Geisser, M.Levine, The $p$-part of K-theory of fields in characteristic $p$, Inv. Math. 139 (2000), 459-494.

[8] T.Geisser, M.Levine, The Bloch-Kato conjecture and a theorem of Suslin-Voevodsky, J.Reine Angew. Math. 530 (2001), 55-103.

[9] H.Gillet, M.Levine, The relative form of Gersten's conjecture over a discrete valuation ring: The smooth case, J. of Pure Appl. Alg. 46 (1987), 59-71.

[10] M.Gros, N.SuwA, La conjecture de Gersten pour les faisceaux de Hodge-Witt logarithmique, Duke Math. J. 57 (1988), 615-628.

[11] K.Kato, Galois cohomology of complete discrete valuation fields, Lecture Notes Math. 967 (1982), 215-238.

[12] K.Kato, S.Saito, Unramified class field theory of arithmetical surfaces. Ann. of Math. 118 (1983), 241-275.

[13] M.Kurihara, A Note on p-adic Etale Cohomology, Proc. Jap. Acad. 63A(1987), 275-278.

[14] M.Levine, Inverting the motivic Bott element, K-theory 19 (2000), 1-28.

[15] M.Levine, Techniques of localization in the theory of algebraic cycles, J. Alg. Geom. 10 (2001), 299-363

[16] M.Levine, K-theory and Motivic Cohomology of Schemes, Preprint 1999

[17] R.MсСавтнY, Relative algebraic K-theory and topological cyclic homology, Acta Math. 179 (1997), 197-222.

[18] J.S.Milne, Motivic cohomology and values of zeta functions, Comp. Math. 68 (1988), 59-102.

[19] J.S.Milne, Etale cohomology, Princeton Math. Series 33

[20] W.Niziol, Crystalline Conjecture via K-theory, Ann. Sci. Math. ENS 31 (1998), 659-682. 
[21] D.Quillen, Higher algebraic K-theory, in: Algebraic $K$-theory, I: Higher $K$-theories (Proc. Conf., Battelle Memorial Inst., Seattle, Wash., 1972), pp. 85-147. Lecture Notes in Math., Vol. 341, Springer, 1973.

[22] K.Sato, An étale Tate twist with finite coefficients and duality in mixed characteristic, Preprint 2002

[23] P.Schneider, p-adic points of motives, Proc. Symp. Pure Math. AMS 55, Part 2 (1994), $225-249$.

[24] N. Spaltenstein, Resolutions of unbounded complexes, Compositio Math. 65 (1988), 121154

[25] A.Susuin, Higher Chow group and étale cohomology, Ann. Math. Studies 132 (2000), 239-554

[26] R.W.Thomason, Algebraic K-theory and etale cohomology, Ann. Sci. ENS 13 (1980), 437552

[27] V.Voevodsky, The 2-part of motivic cohomology, Preprint 2001

University of Southern California, Department of Mathematics, DRB, 1042 W. 36th Place, Los Angeles, CA 90089 